\title{
Post-Basal Insulin Intensification and Healthcare Resource Use in Type 2 Diabetes: A Web-Based Physician Survey in the United States and United Kingdom
}

\author{
Meryl Brod (1) - Amaury Basse $\cdot$ Marie Markert $\cdot$ Kathryn M. Pfeiffer
}

Received: April 15, 2019 / Published online: May 24, 2019

(C) The Author(s) 2019

\begin{abstract}
Introduction: Currently, there is limited knowledge of the healthcare resources and time needed to intensify patients with type 2 diabetes (T2D) treated with basal insulin to more complex treatment regimens. The purpose of the study was to investigate physicians' perspectives on the time and healthcare resources required for post-basal insulin intensification to basal-bolus and to basal in combination with a glucagon-like peptide-1 receptor agonist (GLP1) regimens. The study also examined referrals to specialists for intensification and patient challenges with intensification.
\end{abstract}

Marie Markert's affiliation at the time of the study is given.

Enhanced Digital Features To view enhanced digital features for this article go to https://doi.org/10.6084/ m9.figshare.8117960.

M. Brod $(\bowtie) \cdot$ K. M. Pfeiffer

The Brod Group, 219 Julia Avenue, Mill Valley, CA 94941, USA

e-mail: mbrod@thebrodgroup.net

A. Basse - M. Markert

Novo Nordisk A/S, Vandtaarnsvej 114,

2860 Soeborg, Denmark
Methods: A web-based survey of physicians was conducted in the United Kingdom (UK) and the United States (USA).

Results: A total of 458 physicians completed the survey, including general practitioners $(58.5 \%)$ and specialists (endocrinologists/diabetologists; $41.5 \%$ ). On average, 7.0 healthcare provider (HCP) visits (SD 3.7) over 30.1 weeks (SD 17.4) were required to intensify to a basal-bolus regimen, while 5.7 HCP visits (SD 3.8) over 23.5 weeks (SD 15.2) were needed to intensify to basal insulin in combination with GLP-1. Referral to a specialist for intensification required on average an additional 8 weeks of wait time before intensification. Physicians reported that the complexity of the basal-bolus regimen and frequent injections were key challenges for T2D patients intensifying to basal-bolus, while frequent injections and side effects were key challenges for those intensifying with GLP-1.

Conclusion: Less complex regimens for intensification following basal insulin may help reduce the time and healthcare resources required for intensification and address some of the challenges T2D patients face when intensifying to basal-bolus or basal with GLP-1.

Funding: Novo Nordisk, A/S.

Keywords: Basal-bolus insulin therapy; Diabetes management; Diabetes; GLP-1 receptor agonists; Healthcare resource use; Insulin intensification 


\section{INTRODUCTION}

It is well known that timely treatment intensification and blood glucose control in patients with uncontrolled type 2 diabetes (T2D) are critical to reduce the risks of long-term diabetesrelated complications [1-3]. Yet delays to insulin initiation and treatment intensification in patients with uncontrolled T2D are persistent challenges to diabetes management [4-9]. Postbasal insulin intensification to more complex regimens, such as basal-bolus insulin therapy or basal insulin in combination with a glucagonlike peptide-1 receptor agonist (GLP-1), is particularly challenging. Research estimates that at least $60 \%$ of T2D patients treated with basal insulin alone are uncontrolled based on their hemoglobin A1c (HbA1c) values (HbA1c $\geq 7 \%)$ [10].

The failure to intensify treatment in T2D despite inadequate diabetes control, often referred to as clinical inertia, is a well-documented obstacle to T2D management $[5,6,8,11]$. Both patient and physician factors have been identified as contributing to clinical inertia. Concerns about side effects (e.g., weight gain and hypoglycemia), interference with daily life, beliefs about insulin or diabetes, not wanting to add injections, and relationships with healthcare providers (HCPs) are some of the patient factors that have been associated with reluctance to initiate or intensify treatment in T2D [12-15]. Concerns about hypoglycemia, lack of patient agreement to intensify, patient adherence or compliance to treatment, and views on insulin efficacy have been shown to contribute to clinical inertia in T2D treatment among physicians and other HCPs $[14,15]$.

While the barriers to treatment intensification in T2D are well studied, less is known about the healthcare resources required for intensifying patients treated with basal insulin to more complex regimens, such as basal-bolus insulin therapy or basal insulin in combination with GLP-1. A better understanding of healthcare resource use and time needed for intensification may suggest strategies to prevent delays to postbasal insulin intensification and to improve diabetes control. The purpose of this study was to investigate the process of post-basal insulin intensification for patients with T2D from the perspective of physicians, including general practitioners (GPs) and specialists (endocrinologists/diabetologists), in the United Kingdom (UK) and United States (USA). The study aimed to shed light on the healthcare resource use and time needed for intensification, the process of patient referrals to specialists for intensification, and the concerns and challenges patients may have in relation to intensification.

\section{METHODS}

\section{Survey Development}

A web-based survey was conducted with physicians in the UK and the USA from April to June of 2017. Qualitative analyses of data from concept elicitation focus groups with patients and concept elicitation interviews with physicians informed survey development. Focus groups were conducted with 77 adults diagnosed with type 1 diabetes (T1D) or T2D who were currently treated with basal-bolus insulin therapy. The focus groups were conducted in Germany, the UK, and the USA as part of a previous study and are described in further detail elsewhere [16]. Additionally, 16 physicians in the UK $(n=8)$ and USA $(n=8)$, including both GPs $(n=8)$ and specialists (diabetologists or endocrinologists, $n=8$ ), participated in telephone interviews focused on their clinical experiences with complex regimen treatments for patients with T2D. After survey items were generated, cognitive debriefing interviews were conducted with an additional 12 physicians in the UK and USA to ensure that all instructions and survey items were clear, that the recall periods were appropriate, and that the survey format and structure were acceptable. Prior to survey launch, usability testing was also conducted with an additional 10 physicians in the USA.

The final survey consisted of 91 questions and took approximately $34 \mathrm{~min}$ to complete. Physicians received a modest honorarium (based on fair market rates) upon completion of the survey. All data were self-reported by physicians on the basis of their experiences in 
clinical practice. This survey was administered by a third-party survey research organization. The investigators did not have direct contact with the physicians or access to any potentially identifying information or personal information. On the basis of information from the Office for Human Research Protections at the US Department of Health and Human Services, it was determined that this survey study did not meet the definition of human subjects research and therefore ethics committee approval was not necessary. Informed consent was obtained from all study participants prior to partaking in the survey.

\section{Participants}

Physicians were recruited from proprietary panels of physicians who have opted in to participate in the research panel. The panels are designed to be representative of physicians who care for patients in clinical practice, and all physicians are verified using various administrative databases. Physicians were invited to participate in the survey via email. To be eligible for the survey, physicians had to be a GP or specialist (endocrinologist or diabetologist) with at least 5 years of experience practicing as a physician and at least $40 \%$ of their work time in clinical practice. Additionally, physicians were required to be currently managing at least 10 patients with T2D and have at least four T2D patients treated with a basal-bolus insulin regimen and at least four T2D patients treated with a basal insulin in combination with GLP-1 regimen. In the UK, physicians were required to have at least $80 \%$ of their clinical practice in the National Health Service, as physicians working primarily in private practice may have differing experiences. Physicians in the USA were required to spend at least $80 \%$ of their clinical practice based on fee for service (FFS) patients, as the clinical experiences of physicians who work primarily in a capitated system may differ from those working in an FFS-based system.

\section{Survey Variables}

All physician responses in the survey were selfreported on the basis of physician recall and perceptions. Physicians were not expected to review patient chart data or records when responding to questions. Throughout the survey, physicians were asked to think about average or "example" patients when answering questions so that their responses reflected the experiences of more "typical" or "average" patients rather than patients with particularly difficult or challenging circumstances. "Example" patients were defined as patients aged 35-70 years who have a T2D diagnosis with no major or uncontrolled comorbidities, treated with basal insulin alone (with or without oral antidiabetic agents, OADs), of average cognitive ability/normal mental status, and with a body mass index (BMI) of 25 or greater. This approach of asking physicians to think about more typical or average T2D patients when responding to questions has been employed in previous survey research studies in diabetes [17].

\section{Data Analysis}

Descriptive statistics included frequencies, percentages, means, and standard deviations. Measures of association included comparison of means and cross-tabulations. Significant differences in means between two groups were assessed using Student's $t$ tests. Significant differences in comparisons of categorical variables were assessed using Chi-square tests. For all analyses, the significance level was set at $p<0.05$. Data analysis was conducted using STATA S/E statistical analysis software, version 14 (StataCorp, College Station, TX, USA) [18].

\section{RESULTS}

\section{Sample Descriptive Statistics}

A total of 458 physicians in the UK $(n=225)$ and USA $(n=233)$ completed the survey. GPs comprised $58.5 \%$ of the sample $(n=268)$, while 
Table 1 Physician and practice setting descriptive statistics

\begin{tabular}{|c|c|c|c|c|}
\hline & UK $(n=225)$ & USA $(n=233)$ & Total $(n=458)$ & $p$ \\
\hline \multicolumn{5}{|l|}{ Medical specialty } \\
\hline$n(\%) \mathrm{GP}$ & $137(60.9)$ & $131(56.2)$ & $268(58.5)$ & \multirow[t]{2}{*}{0.311} \\
\hline$n(\%)$ specialist & $88(39.1)$ & $102(43.8)$ & $190(41.5)$ & \\
\hline \multicolumn{5}{|c|}{ Diabetologist or special training in diabetes ${ }^{\mathrm{a} * * *}$} \\
\hline$n(\%)$ yes & $175(77.8)$ & $141(60.5)$ & $316(69.0)$ & $<0.001$ \\
\hline \multicolumn{5}{|l|}{ Years of experience } \\
\hline Mean (SD) & $20.3(7.0)$ & $21.2(7.0)$ & $20.8(7.0)$ & 0.162 \\
\hline \multicolumn{5}{|c|}{ Percentage of time in clinical practice ${ }^{b_{* * *}}$} \\
\hline Mean (SD) & $86.9(10.7)$ & $96.8(5.1)$ & $92.0(9.7)$ & $<0.001$ \\
\hline \multicolumn{5}{|l|}{ Practice setting, $n(\%)$} \\
\hline Private practice ${ }^{\mathrm{a} * * *}$ & $7(3.1)$ & $210(90.1)$ & $217(47.4)$ & $<0.001$ \\
\hline NHS (UK only) & $134(59.6)$ & - & - & - \\
\hline Hospital $^{\mathrm{a}_{* * *}}$ & $83(36.9)$ & $13(5.6)$ & $96(21.0)$ & $<0.001$ \\
\hline Other $^{c}$ & $1(0.4)$ & $10(4.3)$ & $11(2.4)$ & - \\
\hline \multicolumn{5}{|c|}{ Average wait time for new patient appointment (non-urgent) } \\
\hline Mean (SD) weeks & $5.4(4.7)$ & $5.2(4.9)$ & $5.3(4.8)$ & 0.752 \\
\hline \multicolumn{5}{|c|}{ Average wait time for established patient appointment (non-urgent) ${ }^{\mathrm{b}_{* * *}}$} \\
\hline Mean (SD) weeks & $8.3(8.4)$ & $3.5(3.6)$ & $5.8(6.9)$ & $<0.001$ \\
\hline \multicolumn{5}{|c|}{ Percentage of patients seen for $\mathrm{T} 2 \mathrm{D}$ management } \\
\hline Mean (SD) & $36.5(26.5)$ & $40.6(21.1)$ & $38.6(24.0)$ & 0.067 \\
\hline \multicolumn{5}{|c|}{ Number of patients currently managing for $T 2 \mathrm{D}^{\mathrm{b}_{* * *}}$} \\
\hline Mean (SD) & $440.4(391.2)$ & $773.3(693.9)$ & $609.8(589.2)$ & $<0.001$ \\
\hline \multicolumn{5}{|c|}{ Percentage of T2D patients treated with insulin and/or GLP-1 regimen, mean $(S D)^{b, d}$} \\
\hline Basal insulin only*** & $16.7(11.5)$ & $20.7(11.9)$ & $18.7(11.8)$ & $<0.001$ \\
\hline Basal-bolus insulin ${ }^{* *}$ & $13.3(9.9)$ & $17.2(11.9)$ & $15.3(11.1)$ & $<0.001$ \\
\hline Premix insulin*** & $12.5(9.7)$ & $6.8(7.1)$ & $9.6(8.9)$ & $<0.001$ \\
\hline Basal insulin \& GLP-1 & $11.0(7.7)$ & $12.9(8.7)$ & $12.0(8.3)$ & 0.010 \\
\hline \multicolumn{5}{|c|}{ Percentage of T2D patients uncontrolled (based on HbAlc) on treatment regimen, mean $(S D)^{b, d}$} \\
\hline Basal insulin only ${ }^{* * *}$ & $34.3(18.7)$ & $25.5(15.0)$ & $29.8(17.5)$ & $<0.001$ \\
\hline Basal-bolus insulin** & $26.2(17.5)$ & $21.3(14.5)$ & $23.7(16.2)$ & 0.001 \\
\hline Premix insulin ${ }^{\mathrm{e}_{* *}}$ & $31.5(20.0)$ & $26.5(17.4)$ & $29.2(19.0)$ & 0.008 \\
\hline
\end{tabular}


Table 1 continued

\begin{tabular}{|c|c|c|c|c|}
\hline & $\mathrm{UK}(n=225)$ & USA $(n=233)$ & Total $(n=458)$ & $p$ \\
\hline Basal insulin \& GLP-1** & $24.4(17.4)$ & $19.8(16.0)$ & $22.0(16.8)$ & 0.003 \\
\hline \multicolumn{5}{|c|}{$\begin{array}{l}\text { GP general practitioner, } T 2 D \text { type } 2 \text { diabetes, GLP-1 glucagon-like peptide- } 1 \text { receptor agonist, } H b A 1 c \text { hemoglobin } \\
\text { a Chi-square test indicates significant difference by country, }{ }^{* * *} p<0.001 \\
\text { b Student's } t \text { test indicates significant difference by country, }{ }^{* *} p<0.01 \text {; }{ }^{* * *} p<0.001 \\
\text { c Significance test not conducted because of small expected cell count frequencies } \\
\text { d Regimens may be with or without oral antidiabetic agents } \\
\text { e Excludes physicians who reported } 0 \text { patients treated with premix (UK, } n=214 \text {; USA, } n=193 ; \text { total, } n=407 \text { ) }\end{array}$} \\
\hline
\end{tabular}

specialists accounted for $41.5 \%$ of the sample $(n=190)$. Sample descriptive statistics are presented in Table 1.

\section{Healthcare Resource Use and Time to Intensification}

Physician reports on the healthcare resource use and time needed for intensification of "example" T2D patients from basal insulin to basal-bolus and to basal with GLP-1 regimens are presented in Table 2 . On average, the intensification process to a basal-bolus regimen required 7.0 HCP visits (SD 3.7), including visits needed for patients to agree or refuse to intensify, to train patients to intensify, and to titrate patients to their optimal dose after the initial intensification visit. As shown in Table 2, UK physicians reported a significantly greater number of HCP visits for patient training (2.4 visits), on average, compared to US physicians (1.8 visits, $p<0.001)$. Country differences in average HCP visits needed for patients to agree or refuse to intensify and for titration to an optimal dose were not statistically significant.

Average time needed for the entire intensification process to basal-bolus was 30.1 weeks (SD 17.4). UK physicians reported greater patient training time for intensification (8.3 weeks) compared to US physicians (6.4 weeks, $p=0.005$ ), on average. Country differences were not significant for time patients take to agree or refuse to intensify or for the titration process.
For intensification from basal insulin to basal in combination with GLP-1, physicians reported that the intensification process for T2D patients required an average of 5.7 HCP visits (SD 3.8), including visits needed for patients to agree or refuse to intensify, for intensification training, and for titration to an optimal dose after initial intensification. Average HCP visits needed for patients to agree or refuse to intensify and for patient training when intensifying to basal with GLP-1 were similar among physicians in the UK and USA. For titration to an optimal dose, UK physicians reported a slightly lower average number of HCP visits (1.8 visits) compared to US physicians ( 2.0 visits, $p=0.046$ ).

The average total time for the intensification process to basal combined with GLP-1 was 23.5 weeks (SD 15.2). US physicians reported greater time for patients to agree or refuse to intensify (9.3 weeks) compared to UK physicians ( 7.4 weeks, $p=0.004$ ). Country differences in average time for training or titration were not significant.

\section{Referral Process for Intensification in Uncontrolled T2D}

\section{GPs}

On average, GPs reported referring $22.6 \%$ of their uncontrolled (based on HbA1c values) T2D patients to specialists for intensification (SD 25.4). Reported referrals for intensification did not differ significantly by country. The most frequently reported reasons for referring T2D 
Table 2 Average healthcare resource use and time needed to intensify T2D patients treated with basal insulin to basal-bolus and to basal in combination with GLP-1 regimens

\begin{tabular}{|c|c|c|c|c|c|c|c|c|}
\hline & \multicolumn{4}{|c|}{ Intensification to basal-bolus } & \multicolumn{4}{|c|}{ Intensification to basal with GLP-1 } \\
\hline & $\begin{array}{l}\mathrm{UK} \\
(n=215)\end{array}$ & $\begin{array}{l}\text { USA } \\
(n=223)\end{array}$ & $\begin{array}{l}\text { Total } \\
(n=438)\end{array}$ & $p$ & $\begin{array}{l}\mathrm{UK} \\
(n=216)\end{array}$ & $\begin{array}{l}\text { USA } \\
(n=228)\end{array}$ & $\begin{array}{l}\text { Total } \\
(n=444)\end{array}$ & $p$ \\
\hline \multicolumn{9}{|c|}{ Average number of HCP visits needed: mean (SD) } \\
\hline $\begin{array}{l}\text { For patients to agree/ } \\
\text { refuse to intensify }\end{array}$ & $2.4(1.1)$ & $2.3(3.4)$ & $2.3(2.5)$ & 0.657 & $1.9(1.0)$ & $2.1(3.3)$ & $2.0(2.5)$ & 0.418 \\
\hline $\begin{array}{l}\text { To train patients to } \\
\text { intensify }\end{array}$ & $2.4(1.1)$ & $1.8(0.9)$ & $2.1(1.1)$ & $<0.001$ & $1.8(0.9)$ & $1.7(2.3)$ & $1.8(1.7)$ & 0.824 \\
\hline $\begin{array}{l}\text { To titrate patients to } \\
\text { their optimal dose } \\
\text { after the initial } \\
\text { intensification visit }\end{array}$ & $2.6(1.4)$ & $2.5(1.4)$ & $2.6(1.4)$ & 0.457 & $1.8(1.0)$ & $2.0(1.0)$ & $1.9(1.0)$ & 0.046 \\
\hline $\begin{array}{l}\text { Total HCP visits for } \\
\text { intensification }\end{array}$ & $7.4(2.7)$ & $6.6(4.3)$ & $7.0(3.7)$ & 0.012 & $5.5(2.4)$ & $5.9(4.7)$ & $5.7(3.8)$ & 0.337 \\
\hline \multicolumn{9}{|c|}{ Average time needed: mean (SD) weeks } \\
\hline $\begin{array}{l}\text { For patients to agree/ } \\
\text { refuse to intensify }\end{array}$ & $9.5(6.7)$ & $10.4(8.0)$ & $10.0(7.4)$ & 0.200 & $7.4(6.0)$ & $9.3(7.7)$ & $8.4(7.0)$ & 0.004 \\
\hline $\begin{array}{l}\text { To train patients to } \\
\text { intensify }\end{array}$ & $8.3(7.5)$ & $6.4(6.5)$ & $7.3(7.1)$ & 0.005 & $5.3(4.9)$ & $5.3(6.5)$ & $5.3(5.8)$ & 0.892 \\
\hline $\begin{array}{l}\text { To titrate patients to } \\
\text { their optimal dose } \\
\text { after the initial } \\
\text { intensification visit }\end{array}$ & $12.9(8.0)$ & $12.5(7.8)$ & $12.7(7.9)$ & 0.648 & $9.5(6.9)$ & $10.1(6.7)$ & $9.8(6.8)$ & 0.378 \\
\hline $\begin{array}{l}\text { Total time for } \\
\text { intensification }\end{array}$ & 30.7 (17.9) & $29.4(16.9)$ & $30.1(17.4)$ & 0.423 & $22.3(14.0)$ & $24.7(16.1)$ & $23.5(15.2)$ & 0.095 \\
\hline
\end{tabular}

Physicians were asked to report averages based on "typical" T2D patients who are uncontrolled on basal insulin (with or without OADs), with no major or uncontrolled comorbidities. See "Methods" section for additional details $T 2 D$ type 2 diabetes, $G L P-1$ glucagon-like peptide-1 receptor agonist, $H C P$ healthcare provider, $S D$ standard deviation, $O A D$ oral antidiabetes medication

patients to specialists for intensification were being unsuccessful intensifying the patient themselves $(70.8 \%)$, patient requesting the referral $(61.5 \%)$, patient having too many comorbidities $(52.5 \%)$, and complications during treatment $(39.3 \%)$. Following referral, GPs indicated that the average patient wait time before being intensified by a specialist was 8.4 weeks (SD 5.4). Country differences in reasons for referring patients to specialists for intensification are shown in Fig. 1.

After referral to specialists for intensification, GPs reported that $24.6 \%$ of referred patients do not return to the GP's practice for ongoing diabetes care (SD 28.1), while $23.2 \%$ of referred patients return to their practice without having been successfully intensified (SD 21.5), on average. Differences by country were not 


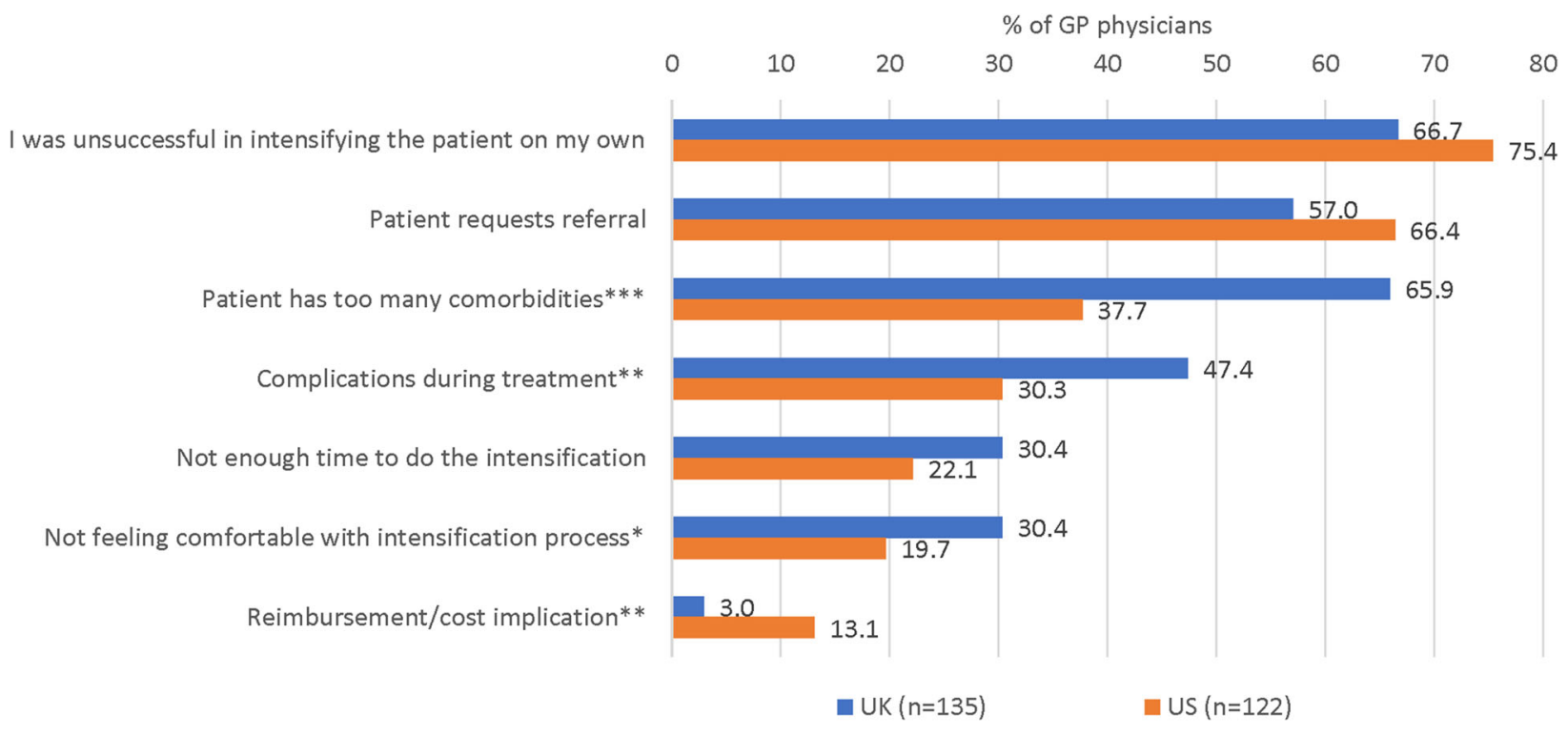

Fig. 1 Reasons GP physicians refer T2D patients to specialists for intensification. Responses not mutually exclusive, $n=257 . T$ tests indicate significant differences by country, ${ }^{*} p<0.05 ;{ }^{* *} p<0.01 ;{ }^{* * *} p<0.001$

statistically significant. The most frequently reported issues (occurring "regularly" or "always") when patients return to the GP's practice after intensification were that patient is not confident that they are following the regimen properly $(23.4 \%)$, patient still requires additional training/information about intensification $(22.2 \%)$, and patient has difficulty titrating on new regimen $(22.2 \%)$. GPs in the UK were significantly more likely to indicate "Patient is not confident that they are following the regimen properly" as an issue (30.4\%) compared to GPs in the USA $(15.6 \%, p=0.018)$.

Among GPs who refer at least some uncontrolled T2D patients to specialists for intensification $(n=257)$, the average time spent on the overall referral process for intensification of T2D patients was $36.7 \mathrm{~min}$ (SD 24.8), including time spent discussing the referral with the patient, following up with the specialist after referral, following up with the patient after referral, and/ or reviewing/assessing a new care plan from the specialist. GPs in the UK reported spending significantly greater time on the referral process (42.3 $\mathrm{min}$ ) compared to those in the USA (30.5 $\min , p<0.001)$.

\section{Specialists}

On average, specialists reported that $68.7 \%$ of their T2D patients were referred to them by GPs for treatment intensification (SD 25.9). UK specialists reported a greater percentage of referred T2D patients $(73.7 \%)$ compared with specialists in the USA $(64.4 \%, p=0.013)$. Average patient wait time for non-urgent appointments with specialists following referral was 8.2 weeks (SD 5.4). Reported wait time was greater among UK specialists (9.3 weeks) compared to specialists in the USA (7.2 weeks, $p=0.008)$. On average, specialists indicated that they received notes or care plans from the referring GPs for $70.1 \%$ of referred patients (SD 31.6). UK specialists reported receiving notes/care plans from referring GPs for more referred patients $(76.8 \%)$ compared to specialists in the USA $(64.3 \%, p=0.006)$.

Specialists reported that $57.7 \%$ of patients referred for basal-bolus intensification continue to see both their referring GP and the specialist (SD 36.6), on average. Specialists in the USA reported a greater percentage of these patients continuing to see both their GP and specialist (71.2\%) compared to specialists in the UK $(42.0 \%, p<0.001)$. For referrals for basal in combination with GLP-1 intensification, 
specialists indicated that $54.0 \%$ of patients continue to see both their GP and specialist (SD 37.4), on average. Specialists in the USA were also more likely to report that these patients continue to see both their GP and specialist (69.8\%) compared to specialists in the UK (35.7\%, $p<0.001)$. Most specialists $(87.9 \%)$ reported spending at least some time preparing notes/care plans for GPs after patients return to their referring GPs, and time spent preparing notes/care plans for GPs did not differ significantly by country.

Specialists indicated that they spend an average of $28.4 \mathrm{~min}$ (SD 21.5) on the overall referral process, including time reviewing notes or care plans from the referring GP and time preparing notes or care plans for the referring GP. Average time specialists spent on the overall referral process did not differ significantly by country.

\section{Patient Experiences With Intensification Process}

Physicians reported on their perspectives of the intensification process for patients treated with basal insulin who require intensification. As described above, physicians were asked to think about more typical or "example" patients when responding to these questions. On average, physicians reported that the target HbA1c value for "example" patients would be $6.9 \%$ (SD 0.5) and that they would consider intensification necessary at an HbA1c value of 7.8\% (SD 0.6).

Among physicians who would intensify at least some "example" patients to basal-bolus, physicians reported that an average of $25.4 \%$ (SD 19.2) of "example" patients refuse to intensify to a basal-bolus regimen, while another $13.9 \%$ (SD 15.4) initially agree but then change their minds $(n=438)$. Likewise, physicians who indicated that they would intensify at least some "example" patients to basal with GLP-1 estimated that an average of $17.6 \%$ (SD 15.2) of "example" patients refuse to intensify to a basal combined with GLP-1 regimen, while another $12.5 \%$ (SD 16.4) initially agree but then change their minds $(n=444)$. Physician-reported reasons that patients refuse to intensify to basal-bolus are shown in Fig. 2, while reported reasons that patients refuse to intensify to basal combined with GLP-1 are displayed in Fig. 3. The frequency of injections required, not wanting to add even one more injection to the current regimen, and feeling that the intensified regimen is too complicated were the most frequently reported reasons for refusal to intensify to basal-bolus. For the basal combined with GLP-1 regimen, physicians reported that not wanting to add even one more injection to the current regimen, negative side effects, and concerns/fear about taking medication after reading package insert were the most frequently reported reasons for patient refusal to intensify to basal insulin in combination with GLP-1.

Physicians also reported on "example" patients' most frequent concerns once intensified. The most frequent concerns of patients intensified to basal-bolus are depicted in Fig. 4, and the most frequent concerns of patients intensified to basal combined with GLP-1 are shown in Fig. 5. For patients intensified to basal-bolus, physicians reported that weight gain was the most frequent concern, followed by experiencing hypoglycemia, and difficulty adhering to the intensified regimen. For patients intensified to basal combined with GLP-1, physicians indicated that insurance coverage/cost, weight loss, and negative side effects were the most frequently expressed concerns.

Among physicians who reported that they would intensify at least some "example" patients to a basal-bolus regimen $(n=438)$, physicians estimated that about $30.3 \%$ of intensified "example" patients report adherence problems (SD 20.2). Physicians were most likely to indicate that patient adherence problems to the basal-bolus regimen were due to the frequency of additional injections (65.7\%), difficulty remembering to take doses $(53.5 \%)$, and feeling that the intensified regimen is too complicated $\quad(53.3 \%) \quad(n=458)$. Following intensification training for basal-bolus, physicians reported that an average of $57.0 \%$ of "example" patients intensified to basal-bolus reach their target HbA1c levels (SD 19.5) and that $37.8 \%$ of "example" patients discontinue the basal-bolus regimen (SD 29.8), on average. 


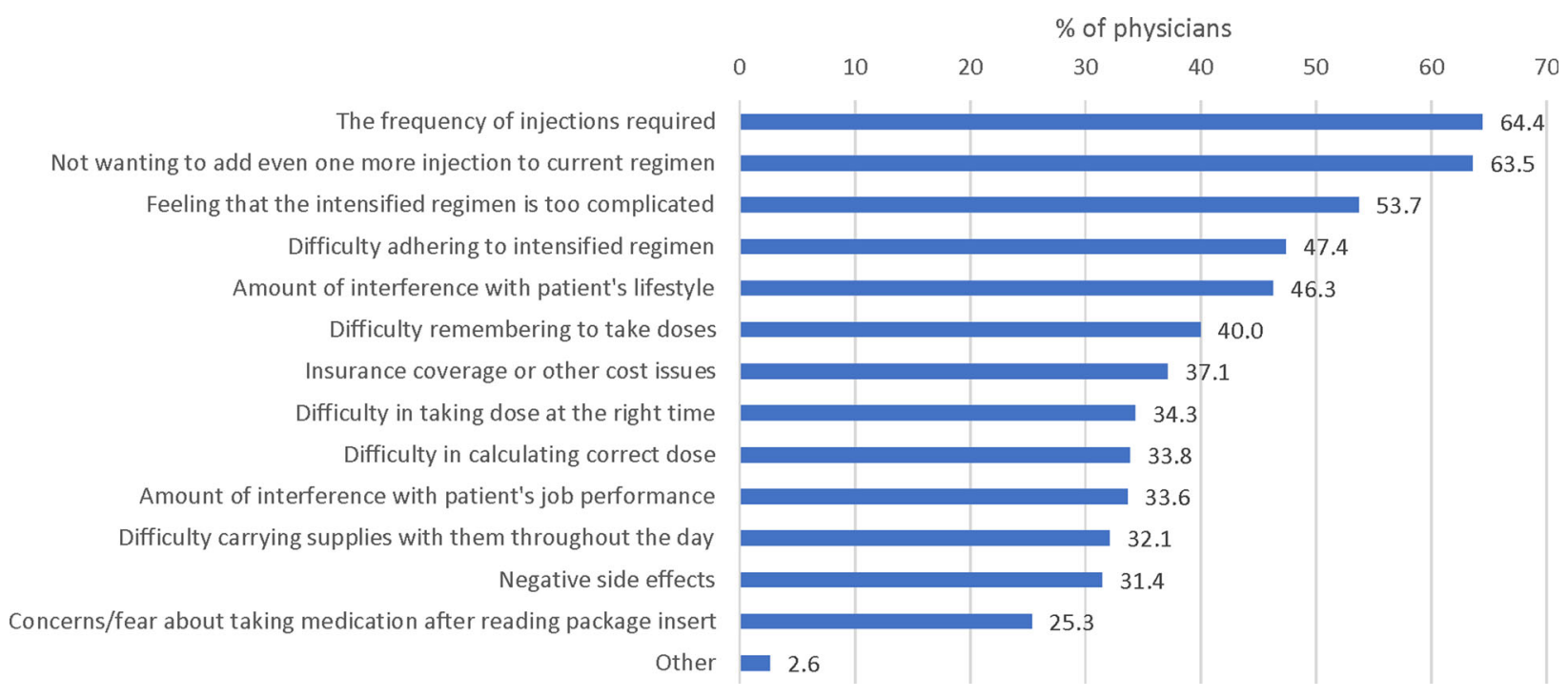

Fig. 2 Reasons patients refuse to intensify to basal-bolus insulin regimen. Responses not mutually exclusive, $n=458$

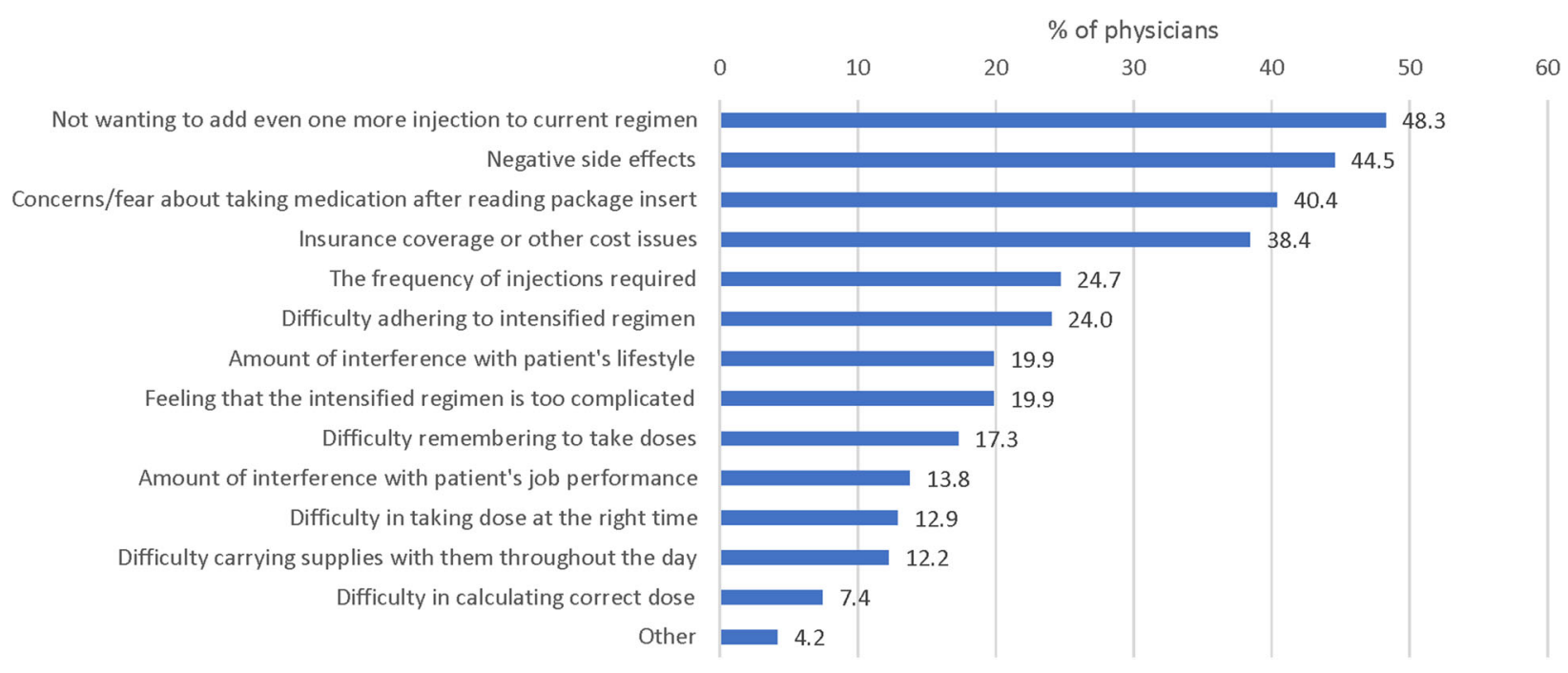

Fig. 3 Reasons patients refuse to intensify to basal insulin in combination with GLP-1. Responses not mutually exclusive, $n=458$

Among physicians who reported that at least some "example" patients discontinue their basal-bolus regimen after intensification $(n=428)$, the most frequently reported reasons for patient discontinuation of their basal-bolus regimen were the frequency of additional injections $(53.0 \%)$, difficulty adhering to the intensified regimen $(50.0 \%)$, and feeling that the intensified regimen is too complicated $(47.0 \%)$.
Among physicians who indicated that they would intensify at least some "example" patients to a basal combined with GLP-1 regimen $(n=444)$, physicians indicated that about $18.6 \%$ of patients report adherence problems (SD 14.3). Physicians most frequently reported that adherence problems to the basal combined with GLP-1 regimen were due to negative side effects $(47.8 \%)$, insurance coverage/cost (38.4\%), and concerns/fears about the 


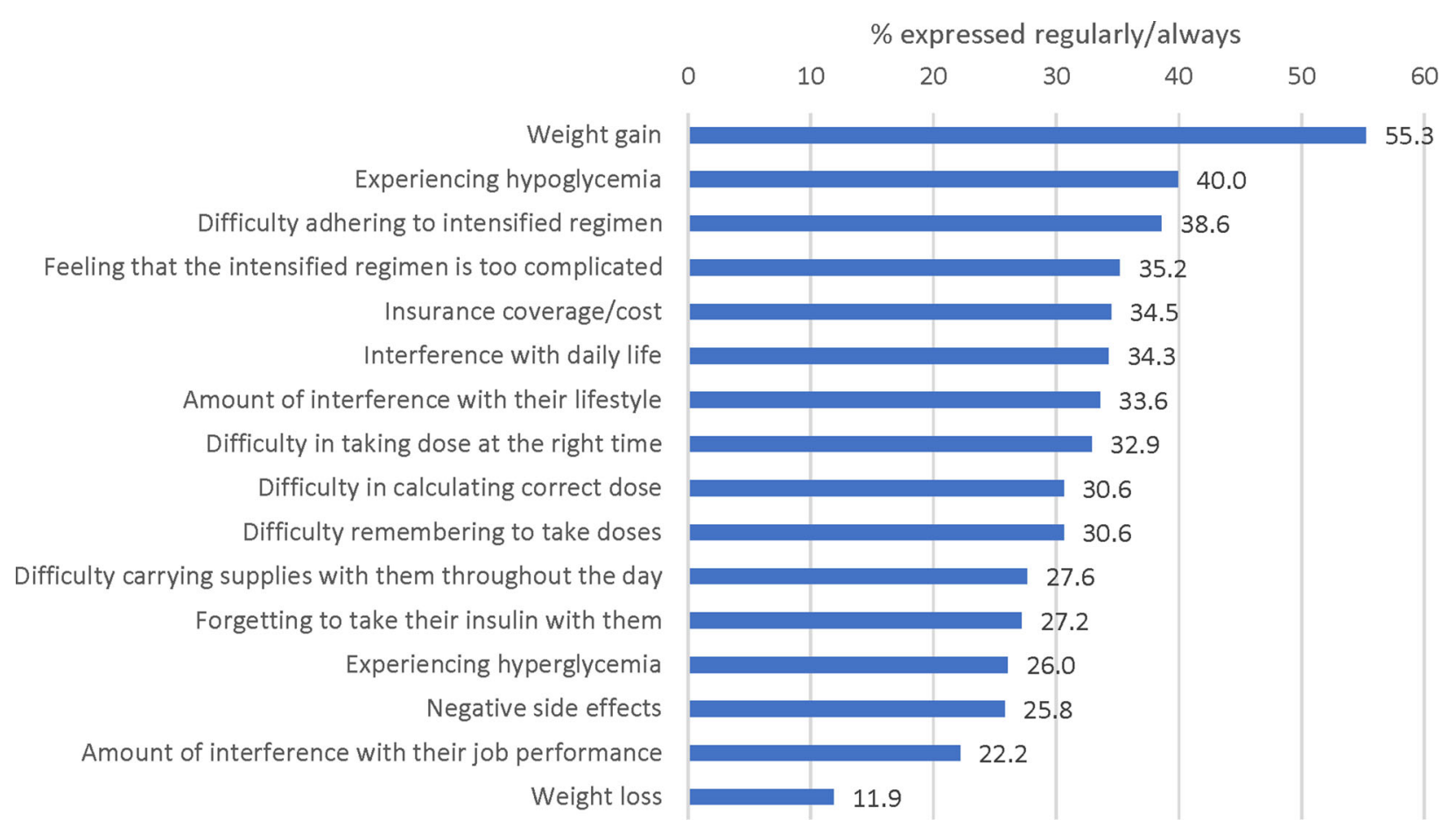

Fig. 4 Concerns expressed by "example" patients intensified to basal-bolus regimen. Responses not mutually exclusive. Question not asked of physicians who reported

that they would not intensify "example" patients to a basal-bolus regimen, $n=438$

medication after reading the package insert $(37.8 \%) \quad(n=458)$. Following intensification training for basal insulin in combination with GLP-1, physicians indicated that $58.5 \%$ of "example" patients reach their target HbA1c levels (SD 20.1) and that 36.7\% of "example" patients discontinue the regimen (SD 30.2), on average. Among physicians who indicated that at least some "example" patients discontinue their basal combined with GLP-1 regimen $(n=427)$, the most frequently reported reasons for patients discontinuing the regimen were negative side effects (55.3\%), followed by patient does not see a response/does not feel that the regimen is working (36.1\%), and insurance coverage/cost (34.2\%).

\section{DISCUSSION}

This study adds to previous research on the intensification process from basal insulin to more complex regimens in T2D patients from the perspective of physicians [17]. Consistent

with prior research $[15,19]$, the findings suggest that the complexity of the basal-bolus regimen, adding additional injections, and negative side effects such as weight gain and hypoglycemia are key patient barriers to intensification to basal-bolus. For intensification to basal in combination with GLP-1, adding additional injections, negative side effects, and insurance coverage/costs were frequently reported reasons for patient refusal to intensify with GLP-1, which is also in line with prior research [19]. Such challenges may result in patient reluctance to intensify, medication non-adherence, and non-persistence, all of which may serve as barriers to timely diabetes control.

While these challenges with intensification to basal-bolus and basal in combination with GLP-1 regimens have been previously studied, this is the first study to look at the healthcare resources and time needed for intensification, as well as the referral process. Results suggest that the intensification process to complex regimens is time-consuming and requires substantial use 


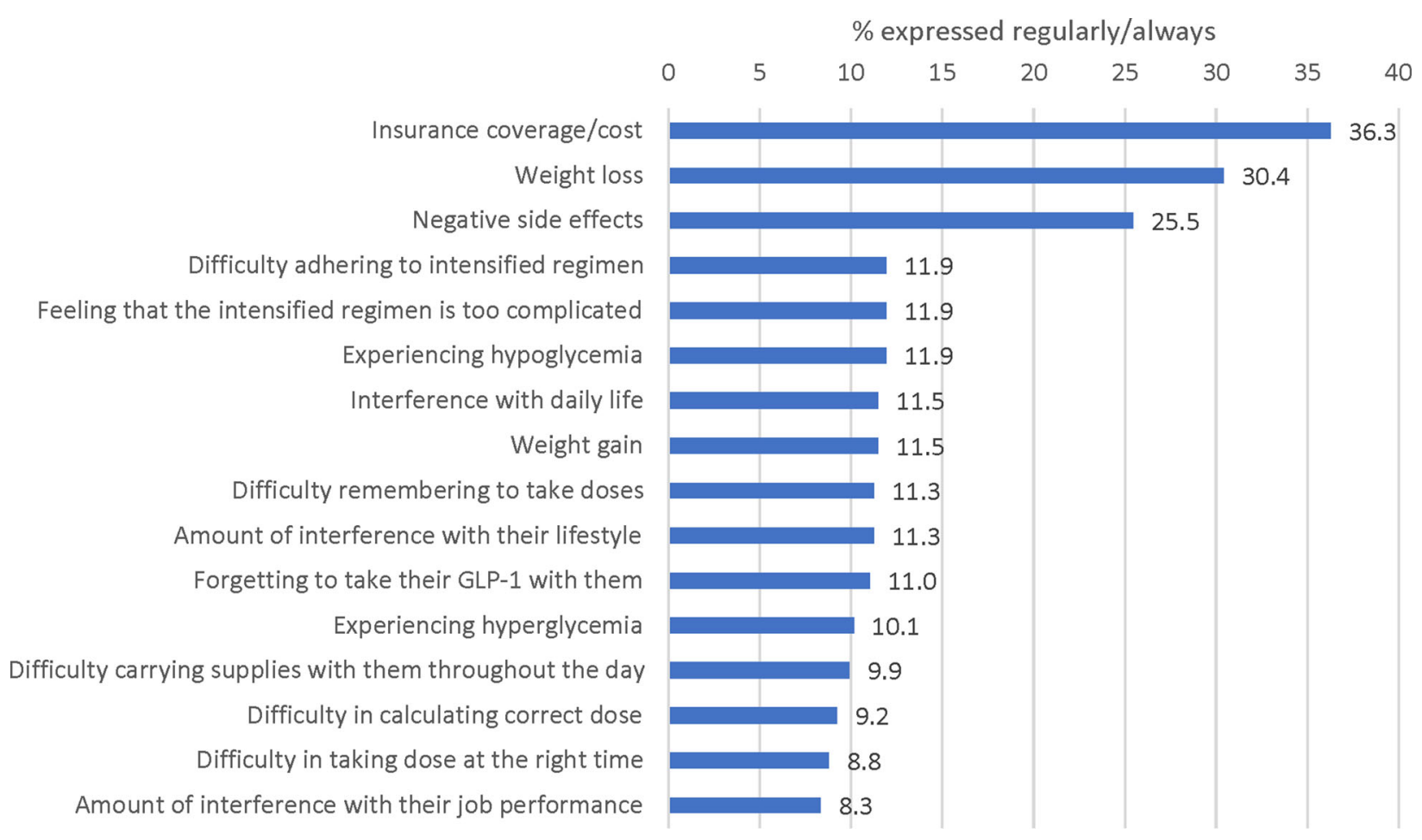

Fig. 5 Concerns expressed by "example” patients intensified to basal insulin in combination with GLP-1 regimen. Responses not mutually exclusive. Question was not asked

of healthcare resources. The basal-bolus intensification process required 7.0 HCP visits, on average, and lasted approximately 30.1 weeks. The intensification process to basal combined with GLP-1 took an average of 5.7 HCP visits and about 23.5 weeks. The relatively high number of HCP visits and length of time for intensification with GLP-1 are somewhat surprising given that intensifying with GLP-1 is theoretically less complex than intensifying to basal-bolus. The substantial use of healthcare resources for intensification have cost implications and may pose financial burdens on both patients and payers.

Additionally, the lengthy time to treatment intensification suggests that T2D patients may experience long periods of time in which their diabetes is uncontrolled before their regimen is fully intensified. When referral to a specialist is required for intensification, the wait time for an appointment adds an average of 8.4 additional weeks to the intensification process. This means of physicians who reported that they would not intensify "example" patients to a basal insulin in combination with GLP-1 regimen, $n=444$

an average intensification time of 38.5 weeks (approximately 9 months) for basal-bolus therapy and 31.9 weeks (approximately 8 months) for intensification to basal insulin in combination with GLP-1. Such delays to post-basal insulin intensification are concerning given the importance of timely blood glucose control to reduce risks of long-term diabetes-related complications [1, 3]. Even when T2D patients are intensified successfully to basal-bolus therapy or basal insulin in combination with GLP-1, physicians reported that medication non-adherence and non-persistence were frequent problems and that some intensified patients do not reach their HbA1c targets, all of which are well-known challenges to T2D management [20-22].

In sum, the study findings suggest that more simplified treatment options for post-basal insulin intensification may help reduce the time and healthcare resources required for intensification. Additionally, the results suggest 
that less complex treatments would also address patient concerns related to frequent injections and regimen complexity, which may improve medication adherence and persistence, and ultimately improve diabetes control. Less complex treatment options may also reduce the need for referrals to specialists for intensification. Recent research suggests that a new treatment option for post-basal insulin intensification, which includes a less complex regimen and fewer injections compared to basal-bolus therapy or basal insulin in combination with GLP-1, reduces the time and healthcare resources necessary for intensification [17]. Further, patient and physician education may also help reduce the number of HCP visits and time required for post-basal insulin intensification.

Several study limitations should be considered when interpreting results. The reliance on physician recall of experiences and perceptions, rather than a systematic review of chart data, means there is the potential for recall bias. Moreover, asking physicians to report on "example" patients with more "typical" or "average" experiences does not capture the broad range of intensification experiences that patients may have. A systematic review of chart data may provide more robust and complete information on the post-basal insulin intensification process in T2D.

Although the physician panels used to recruit physicians for the survey were designed to be representative of physicians in each country, the possibility of selection bias also cannot be ruled out. Further, some overlap may exist in the reported time and healthcare resources needed for intensification training and titration. Although the survey questions were designed to avoid such overlap, it is possible that some HCP visits include continued training, as well as titration. Finally, results may not be generalizable to other countries or in differing healthcare systems outside of the UK and USA. Future research might investigate how the healthcare resources and time required for post-basal insulin intensification, as well as the referral process, may differ in other healthcare systems.

\section{CONCLUSION}

Intensifying T2D patients treated with basal insulin to more complex regimens, including basal-bolus insulin therapy and basal insulin in combination with GLP-1, is a time-consuming process and requires substantial use of healthcare resources. The findings have important implications for healthcare costs to patients and payers, as well as costs related to the length of time T2D patients remain uncontrolled during the intensification process. The results suggest that additional physician and patient education may be warranted to reduce intensification time and thereby reduce that time that $\mathrm{T} 2 \mathrm{D}$ patients remain poorly controlled. The findings also suggest that less complex treatment regimens may help reduce both the costs and time needed for post-basal insulin intensification in T2D.

\section{ACKNOWLEDGEMENTS}

The authors would like to thank the study participants for their time and effort in completing the survey.

Funding. Sponsorship for this study and the article processing charges were funded by Novo Nordisk, A/S. All authors had full access to all of the data in this study and take complete responsibility for the integrity of the data and accuracy of the data analysis.

Editorial Assistance. Jane Beck of The Brod Group provided editorial assistance, which was included in the support provided by Novo Nordisk, A/S.

Authorship. All named authors meet the International Committee of Medical Journal Editors (ICMJE) criteria for authorship for this article, take responsibility for the integrity of the work as a whole, and have given their approval for this version to be published.

Prior Presentations. Some material in this paper was presented in two posters entitled 
"The costs of intensification from basal insulin to basal-bolus in type 2 diabetes: a survey of physicians" (Poster ID: PDB44) and "The costs of intensifying type 2 diabetes patients treated with basal insulin to basal in combination with GLP-1: a physician survey" (Poster ID: PDB43) presented at the 23rd Annual International Meeting of the International Society for Pharmacoeconomics and Outcomes Research (ISPOR), 19-23 May 2018, Baltimore, MD, USA.

Disclosures. Meryl Brod is a paid consultant to the pharmaceutical industry, including Novo Nordisk, A/S. Kathryn M. Pfeiffer is a paid consultant to the pharmaceutical industry, including Novo Nordisk, A/S. Amaury Basse is an employee of Novo Nordisk, A/S. Marie Markert was an employee of Novo Nordisk, A/S at the time of the study and is currently an employee of Ferring Pharmaceuticals.

Compliance with Ethics Guidelines. This survey was administered by a third-party survey research organization. The investigators did not have direct contact with the physicians or access to any potentially identifying information or personal information. On the basis of information from the Office for Human Research Protections at the US Department of Health and Human Services, it was determined that this survey study did not meet the definition of human subjects research and therefore ethics committee approval was not necessary. Informed consent was obtained from all study participants prior to partaking in the survey.

Data Availability. The subject-level analysis data sets for the research presented in the publication are available from the corresponding author, $\mathrm{MB}$, on reasonable request.

Open Access. This article is distributed under the terms of the Creative Commons Attribution-NonCommercial 4.0 International License (http://creativecommons.org/licenses/ by-nc/4.0/), which permits any noncommercial use, distribution, and reproduction in any medium, provided you give appropriate credit to the original author(s) and the source, provide a link to the Creative Commons license, and indicate if changes were made.

\section{REFERENCES}

1. Lovre D, Fonseca V. Benefits of timely basal insulin control in patients with type 2 diabetes. J Diabetes Complic. 2015;29(2):295-301.

2. Inzucchi SE, Bergenstal RM, Buse JB, et al. Management of hyperglycemia in type 2 diabetes, 2015: a patient-centered approach: update to a position statement of the American Diabetes Association and the European Association for the Study of Diabetes. Diabetes Care. 2015;38(1):140-9.

3. American Diabetes Association. Standards of medical care in diabetes-2015. Diabetes Care. 2015;38(Suppl 1):S1-93.

4. Blak BT, Smith HT, Hards M, Maguire A, Gimeno V. A retrospective database study of insulin initiation in patients with type 2 diabetes in UK primary care. Diabet Med. 2012;29(8):e191-8.

5. Khunti K, Nikolajsen A, Thorsted BL, Andersen M, Davies MJ, Paul SK. Clinical inertia with regard to intensifying therapy in people with type 2 diabetes treated with basal insulin. Diabetes Obes Metab. 2016;18(4):401-9.

6. Khunti K, Wolden ML, Thorsted BL, Andersen M, Davies MJ. Clinical inertia in people with type 2 diabetes: a retrospective cohort study of more than 80,000 people. Diabetes Care. 2013;36(11):3411-7.

7. Nichols GA, Koo YH, Shah SN. Delay of insulin addition to oral combination therapy despite inadequate glycemic control: delay of insulin therapy. J Gen Intern Med. 2007;22(4):453-8.

8. Shah BR, Hux JE, Laupacis A, Zinman B, Van Walraven $C$. Clinical inertia in response to inadequate glycemic control: do specialists differ from primary care physicians? Diabetes Care. 2005;28(3):600-6.

9. Ross SA, Tildesley HD, Ashkenas J. Barriers to effective insulin treatment: the persistence of poor glycemic control in type 2 diabetes. Curr Med Res Opin. 2011;27(sup3):13-20.

10. Giugliano D, Maiorino MI, Bellastella G, Chiodini $\mathrm{P}$, Ceriello A, Esposito K. Efficacy of insulin analogs in achieving the hemoglobin A1c target of $<7 \%$ in type 2 diabetes: meta-analysis of randomized controlled trials. Diabetes Care. 2011;34(2):510-7. 
11. Khunti K, Gomes MB, Pocock S, et al. Therapeutic inertia in the treatment of hyperglycaemia in patients with type 2 diabetes: a systematic review. Diabetes Obes Metab. 2018;20(2):427-37.

12. Brod M, Kongso JH, Lessard S, Christensen TL. Psychological insulin resistance: patient beliefs and implications for diabetes management. Qual Life Res. 2009;18(1):23-32.

13. Nam S, Chesla C, Stotts NA, Kroon L, Janson SL. Factors associated with psychological insulin resistance in individuals with type 2 diabetes. Diabetes Care. 2010;33(8):1747-9.

14. Peyrot M, Rubin RR, Lauritzen T, Skovlund SE, Snoek FJ, Matthews DR, et al. Resistance to insulin therapy among patients and providers: results of the cross-national Diabetes Attitudes, Wishes, and Needs (DAWN) study. Diabetes Care. 2005;28(11): 2673-9.

15. Brod M, Pfeiffer KM, Barnett AH, Berntorp K, Vilsboll T, Weissenberger B. Perceptions of diabetes control among physicians and people with type 2 diabetes uncontrolled on basal insulin in Sweden, Switzerland, and the United Kingdom. Curr Med Res Opin. 2016;32(6):981-9.

16. Brod M, Nikolajsen A, Weatherall J, Pfeiffer KM. The economic burden of post-prandial hyperglycemia (PPH) among people with type 1 and type 2 diabetes in three countries. Diabetes Ther. 2016;7(1):75-90.
17. Drummond R, Baru A, Dutkiewicz M, Basse A, Tengmark B-O. Physicians' real-world experience with IDegLira: results of a European survey. BMJ Open Diabetes Res Care. 2018;6(1):e000531.

18. StataCorp. Stata Statistical Software: Release 14. College Station, TX: StataCorp LP; 2015.

19. Pfeiffer KM, Basse A, Lee XY, Waldman LT. Diabetes management and healthcare resource use when intensifying from basal insulin to basal-bolus: a survey of type 2 diabetes patients. Diabetes Therapy. 2018;9(5):1931-44.

20. Peyrot M, Barnett AH, Meneghini LF, SchummDraeger PM. Factors associated with injection omission/non-adherence in the Global Attitudes of Patients and Physicians in Insulin Therapy study. Diabetes Obes Metab. 2012;14(12):1081-7.

21. Garcia-Perez LE, Alvarez M, Dilla T, Gil-Guillen V, Orozco-Beltran D. Adherence to therapies in patients with type 2 diabetes. Diabetes Ther. 2013;4(2):175-94.

22. Peyrot M, Barnett AH, Meneghini LF, SchummDraeger PM. Insulin adherence behaviours and barriers in the multinational global attitudes of patients and physicians in insulin therapy study. Diabet Med. 2012;29(5):682-9. 